\title{
NATURAL CONVECTION HEAT TRANSFER OSCILLATORY FLOW OF AN ELASTICO-VISCOUS FLUID FROM VERTICAL PLATE
}

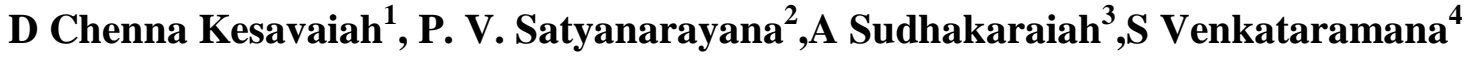 \\ ${ }^{1}$ Assistant Professor, Department of $H \& B S$, Visvesvaraya College of Engineering \& Technology, \\ Greater Hyderabad, A.P, India, ${ }^{2}$ Assistant Professor (Senior), Fluid Dynamics Division, School of Advanced Sciences, VIT \\ University, Vellore., ${ }^{3}$ Assistant Professor, Department of Future Studies, Sri Venkateswara University, Tirupati - 517 \\ 502, A.P, India, ${ }^{4}$ Professor, Department of Mathematics, Sri Venkateswara University, Tirupati - 517 502, A.P, India \\ chennakesavaiah@gmail.com,pvsatya8@yahoo.co.in,Sudha.maths@rediffmail.com
}

\begin{abstract}
This paper examines the problem of two dimensional unsteady free convection oscillatory flow of an Elastico-viscous fluid past an impulsively started infinite vertical plate with constant heat flux and heat generation. The flow is subjected to a constant suction. Using perturbation techniquethe expressions are obtained for velocity, temperature distribution and skin friction. The results obtained are discussed for various numerical values of the parameters entered into the equations governing the flow, with help of graphs.
\end{abstract}

Index terms:Elastico-viscous fluid, free convection, Porous medium, vertical plate.

\section{INTRODUCTION}

The boundary layer phenomenon in non-Newtonian fluids was studied by a few research workers, whose constitutive equations are characterized by Walters [22], exhibits a boundary layer phenomenon. The effect of unsteady fluctuations of the free velocity on the flow in the boundary layer of an incompressible elastico-viscous liquid [Walters fluid (model $\left.\mathrm{B}^{\prime}\right)$ ] past an infinite porous plate with constant suction velocity was discussed by Kaloni [8]. Flow and heat transfer in the boundary layer on a continuous moving surface was considered by Tsouet. al.[20]. Vajravelu [21] studied the solution of boundary layer flow and heat transfer over a continuous porous surface moving in an oscillating free stream. Unsteady free convection flow of an incompressible fluid along a moving surface in an oscillating free stream was presented by Sharma and Gocher [15].

Boundary layer behavior over a continuous moving surface is an important type of flow occurring in several engineering processes, for example materials manufactured by extrusion processes and heat treated materials traveling between a feed roll and a wind up roll or on conveyor belts possesses the characteristics of a moving continuous surface. In view of these applications, Sakiadis [14] initiated the study of boundary layer flow over a continuous solid surface moving with constant speed. Due to entrainment of ambient fluid, this boundary layer flow is quite different than boundary layer flow over a semi-infinite flat plate. Erickson et. al. [6] extended this problem to the case in which the transverse velocity at the moving surface is non zero, with heat and mass transfer in the boundary layer being taken into account. Beg et al. [1] have presented an analysis on unsteady natural convection flow past an infinite vertical plate embedded within a highly porous medium under the influence of uniform magnetic field, wherein, the suction velocity is subjected to small amplitude oscillations in time about the steady non-zero mean suction velocity.

The unsteady free convective flow of an elastico-viscous fluid was studied by Soundalgekar [18]. Revankar et al [13], Singh [16] discussed the flow of elastico-viscous fluid past impulsively started plate. Hayat et al. [7] discussed the flow of visco-elastic fluid past an oscillating plate, in which he discussed the influence of suction/injection on velocity distribution. In many problems, particularly those involving the cooling of electrical and nuclear component, the wall heat flux is specified. In such problem overheating burn out and melt down are very important issues. The problems with prescribed heat flux are special case of the vast analytical accessible class of problem. Lee et al. [9], Malarvizhi et al. [11], Burak et al. [2],Kolani [8] and Pantokraters [12] are some of the researchers who have investigated the convection flow with prescribed heat flux condition. Effects of the chemical reaction and radiation absorption on free convection flow through porous medium with variable suction in the presence of uniform magnetic field were studied by SudheerBabu and Satyanarayana [19]. Devikaet.al [5]MHD Oscillatory flow of a Visco Elastic fluid in a Porous channel with chemical Reaction Singh [17]Exact solution of an oscillatory MHD flow in a channel filled with porous medium. Chenna Kesavaiah and Sudhakaraiah [4]A note on heat transfer to magnetic field Oscillatory flow of a visco-elastic fluid. 
Therefore, the objective of the present investigation in to study the two dimensional unsteady free convection oscillatory flow of an elastico-viscous fluid past an impulsively started infinite vertical plate in the presence of constant suction, heat flux and heat generation. The effects of elastic parameter on velocities and temperature fluids are discussed in detail. Also the results are in very good agreement with the results of Chaudhary [3] in the absence of heat absorption.

\section{MATHEMATICAL ANALYSIS}

We consider a two dimensional, unsteady free convection flow of an elastico-viscous fluid past a steadily moving infinite porous plate with constant suction, heat flux and heat generation. In Cartesian co-ordinate system the $x^{\prime}$ axis taken along the plate in the upward direction, being the direction of the flow and $y^{\prime}$ axis is taken normal to the plate. Let the component of velocity $u^{\prime}, v^{\prime}$ along $x^{\prime}, y^{\prime}$ directions respectively. Under these assumptions the appropriate governing equations of continuity momentum and energy are given by

$$
\frac{\partial \mathrm{v}^{\prime}}{\partial y^{\prime}}=0
$$

$$
\begin{aligned}
\frac{\partial u^{\prime}}{\partial t^{\prime}}+\mathrm{v}^{\prime} \frac{\partial u^{\prime}}{\partial y^{\prime}} & =\frac{\partial U^{\prime}}{\partial t^{\prime}}+g \beta\left(T^{\prime}-T_{\infty}^{\prime}\right)+v \frac{\partial^{2} u^{\prime}}{\partial y^{\prime 2}} \\
& -\frac{k}{\rho}\left(\frac{\partial^{3} u^{\prime}}{\partial y^{\prime 2} \partial t^{\prime}}+\mathrm{v}^{\prime} \frac{\partial^{3} u^{\prime}}{\partial y^{\prime 3}}\right)
\end{aligned}
$$

$$
\begin{aligned}
\frac{\partial T^{\prime}}{\partial t^{\prime}}+\mathrm{v}^{\prime} \frac{\partial T^{\prime}}{\partial y^{\prime}} & =\frac{\lambda}{\rho c_{p}} \frac{\partial^{2} T^{\prime}}{\partial y^{\prime 2}}+\frac{v}{c_{p}}\left(\frac{\partial u^{\prime}}{\partial y^{\prime}}\right)^{2} \\
& -\frac{Q_{0}}{\rho c_{p}}\left(T^{\prime}-T_{\infty}^{\prime}\right)
\end{aligned}
$$

The boundary conditions are

For $t^{\prime} \leq 0 \quad u^{\prime}\left(y^{\prime}, t^{\prime}\right)=0, T^{\prime}\left(y^{\prime}, t^{\prime}\right)=T_{\infty}^{\prime}$

$$
\text { For } \begin{array}{r}
t^{\prime} \geq 0 \quad u\left(0, t^{\prime}\right)=U_{0},\left(\frac{\partial T^{\prime}}{\partial y^{\prime}}\right)=-\frac{q^{\prime}}{\lambda} \\
u^{\prime}\left(\infty, t^{\prime}\right)=U^{\prime}\left(t^{\prime}\right), T^{\prime}\left(\infty, t^{\prime}\right)=T_{\infty}^{\prime}
\end{array}
$$

Where $U_{0}$ is the constant velocity of moving porous plate and $T_{\infty}^{\prime}$ is its temperature, $q$ is the heat flux per unit area, per unit time.
The continuity equation (1) gives

$\mathrm{v}^{\prime}=-\mathrm{v}_{0}$

Where $\mathrm{v}_{0}(>0)$ is suction velocity of the fluid through the porous plate.

Where $t^{\prime}$ is time, $u^{\prime}, \mathrm{v}^{\prime}$ are the velocity components along and perpendicular to the porous plate, $g$ acceleration due to gravity, $T^{\prime}$ the fluid temperature inside the thermal boundary layer, $T_{\infty}^{\prime}$ temperature away to the porous plate, $v_{\text {kinematic }}$ viscosity, $\mathrm{k}$ the elastic parameter, $\lambda$ the thermal conductivity of the plate, $c_{p}$ the specific heat of the fluid under constant pressure, $\beta$ the coefficient of volumetric expansion of the fluid, $\mathrm{U}$ is the free stream velocity, $\mathrm{k}$ is Elastic parameter, $T_{w}^{\prime}$ is Constant velocity of moving porous plate temperature, U0is the constant velocity of moving porous plate, v0 the suction velocity of the fluid through the porous plate, $v$ is kinematic viscosity, $\rho$ density of the fluid, $\theta$ is nondimensional temperature, $\beta$ is coefficient of volumetric expansion of the fluid.

Now in order to non-dimensionalize equations (2) and (3) we introduce the following non-dimensional quantities

$$
\begin{aligned}
& y=\frac{y^{\prime} \mathrm{v}_{0}}{v}, \quad t=\frac{t^{\prime} \mathrm{v}_{0}^{2}}{v}, \quad u=\frac{u^{\prime}}{U_{0}}, U=\frac{U^{\prime}}{U_{0}} \\
& \theta=\frac{\left(T^{\prime}-T_{\infty}^{\prime}\right) \lambda \mathrm{v}_{0}}{q^{\prime} v}, P=\frac{\mu c_{p}}{\lambda}, \phi=\frac{v Q_{0}}{\rho c_{p} \mathrm{v}_{0}^{2}} \\
& k=\frac{k^{\prime} \mathrm{v}_{0}^{2}}{\rho v^{2}}, \quad E=\frac{\lambda \mathrm{v}_{0} U_{0}^{2}}{q^{\prime} v c_{p}}, \quad G=\frac{g \beta q^{\prime} v^{2}}{\lambda \mathrm{v}_{0}{ }^{3} U_{0}}
\end{aligned}
$$

In view of above non-dimensional quantities, equations (2) and (3) becomes

$$
\begin{aligned}
& \frac{\partial u}{\partial t}-\frac{\partial u}{\partial y}=\frac{\partial U}{\partial t}+G \theta+\frac{\partial^{2} u}{\partial y^{2}}-k\left(\frac{\partial^{3} u}{\partial y^{2} \partial t}+\frac{\partial^{3} u}{\partial y^{3}}\right) \\
& \frac{\partial \theta}{\partial t}-\frac{\partial \theta}{\partial y}=\frac{1}{P} \frac{\partial^{2} \theta}{\partial y^{2}}+E\left(\frac{\partial u}{\partial y}\right)^{2}-\phi \theta
\end{aligned}
$$

The corresponding boundary conditions are 
$y=0, u=1, \frac{\partial \theta}{\partial y}=-1$

$y \rightarrow \infty, \theta \rightarrow 0, u \rightarrow U(t)$

wherek is Elastic parameter, PrPrandtl number, Q The heat flux per unit area, per unit time, $Q_{0}$ Dimensional Heat absorption coefficient, $\phi$ Heat source parameter

In order to solve coupled nonlinear systems, we assume that the following Lighthill[10], i.e., there exist a mean steady flow and it is superimposed on the unsteady oscillatory flow. Then, in the neighborhood of the plate, we represent the velocity and temperature fields as

$\left.\begin{array}{l}u(y, t)=u_{0}(y)+\varepsilon u_{1}(y) e^{\mathrm{int}} \\ \theta(y, t)=\theta_{0}(y)+\varepsilon \theta_{1}(y) e^{\mathrm{int}}\end{array}\right\}$

and for free stream

$U(t)=1+\varepsilon e^{\mathrm{int}}$

where $\mathcal{E}$ is a small constant quantity $\ll<1$.

Putting equation (10) in (7) and (8) and equating like powers and neglecting coefficient of $\varepsilon^{2}$, we get

$k u_{0}^{\prime \prime \prime}-u_{0}^{\prime \prime}-u_{0}^{\prime}=G \theta_{0}$

$k u_{1}^{\prime \prime \prime}-(1-k i n) u_{1}^{\prime \prime}-u_{1}^{\prime}+i n u_{1}=i n+G \theta_{1}$

$\theta_{0}^{\prime \prime}+P \theta_{0}^{\prime}-P \phi \theta_{0}=-P E u_{0}^{\prime 2}$

$\theta_{1}^{\prime \prime}+P \theta_{1}^{\prime}-($ in $-\phi) P \theta_{1}=-2 P E u_{0}^{\prime} u_{1}^{\prime}$

where prime denotes differentiation with respect to $\mathrm{y}$.

Corresponding boundary conditions are

$$
\begin{aligned}
& y=0, u_{0}=1, u_{1}=0, \frac{\partial \theta_{0}}{\partial y}=-1, \frac{\partial \theta_{1}}{\partial y}=0 \\
& y \rightarrow \infty, u_{0} \rightarrow 1, u_{1} \rightarrow 1, \theta_{0} \rightarrow 0, \theta_{1} \rightarrow 0
\end{aligned}
$$

Now, equations (11) and (12) are third-order differential equations when $k \neq 0$ and for $k=0_{\text {they reduced to }}$ equations governing the Newtonian fluid. Hence, the presence of the elasticity of the fluid increases the order of the governing equations from two to three and therefore, they need three boundary conditions for their unique solution. But there are prescribed only two boundary conditions in equation (15). Therefore for a unique solution, we follow Beard and Walters [22] and assume that the solution in the form

$$
\begin{aligned}
& u_{i}(y)=u_{i 1}(y)+k u_{i 2}(y)+0\left(k^{2}\right) \\
& \theta_{i}(y)=\theta_{i 1}(y)+k \theta_{i 2}(y)+0\left(k^{2}\right)
\end{aligned}
$$

Where $u_{i}, \theta_{i}(i=0,1)$ are the mean $(i=0)$ and the fluctuating $(i=1)$ parts of velocity and temperature respectively, as $k$ is small for elastico-viscous fluid equation (16) is valid. Introducing (16) into (11)-(14), we get

$u_{01}^{\prime \prime}+u_{01}^{\prime}=-G \theta_{01}$

$u_{02}^{\prime \prime}+u_{02}^{\prime}=-G \theta_{02}+u_{01}^{\prime \prime \prime}$

$u_{11}^{\prime \prime}+u_{11}^{\prime}-i n u_{11}=-i n-G \theta_{11}$

$u_{12}^{\prime \prime}+u_{12}^{\prime}-i n u_{12}=-G \theta_{12}+u_{11}^{\prime \prime \prime}+i n u_{11}^{\prime \prime}$

$\theta_{01}^{\prime \prime}+P \theta_{01}^{\prime}-P \phi \theta_{01}=-P E u_{01}^{\prime 2}$

$\theta_{02}^{\prime \prime}+P \theta_{02}^{\prime}-P \phi \theta_{02}=-2 P E u_{01}^{\prime} u_{02}^{\prime}$

$\theta_{11}^{\prime \prime}+P \theta_{11}^{\prime}-($ in $-\phi) P \theta_{11}=-2 P E u_{01}^{\prime} u_{11}^{\prime}$

$\theta_{12}^{\prime \prime}+P \theta_{12}^{\prime}-($ in $-\phi) P \theta_{12}=$

$$
-2 P E\left(u_{01}^{\prime} u_{12}^{\prime}+u_{11}^{\prime} u_{02}^{\prime}\right)
$$

and the corresponding boundary conditions are

$$
y=0\left\{\begin{array}{l}
u_{01}=1, u_{02}=0=u_{11}=u_{12} \\
\theta_{01}{ }^{\prime}=-1, \theta_{02}{ }^{\prime}=\theta_{11}{ }^{\prime}=\theta_{12}{ }^{\prime}=0
\end{array}\right.
$$

where dash denotes differentiation with respect to $\mathrm{y}$. 
$y \rightarrow \infty\left\{\begin{array}{l}u_{01} \rightarrow 1, u_{02} \rightarrow 0, u_{11} \rightarrow 1, u_{12} \rightarrow 0 \\ \theta_{01} \rightarrow 0, \theta_{02} \rightarrow 0, \theta_{11} \rightarrow 0, \theta_{12} \rightarrow 0\end{array}\right.$

The system of equations (17) - (24) are still coupled nonlinear, we can assume that

$u_{01}=u_{011}+E u_{012}, u_{02}=u_{021}+E u_{022}$

$u_{11}=u_{111}+E u_{112}, u_{12}=u_{121}+E u_{122}$

$\theta_{01}=\theta_{011}+E \theta_{012}, \theta_{02}=\theta_{021}+E \theta_{022}$

$\theta_{11}=\theta_{111}+E \theta_{112}, \theta_{12}=\theta_{121}+E \theta_{122}$

where is $E$ Eckert number and very small for an incompressible fluid.

Substituting equation (26) in (17) - (24), equating the coefficient of different powers of $E$ and neglecting coefficient of $E^{2}$ and using equation (25) we get

$$
\begin{aligned}
& u_{011}^{\prime \prime}+u_{011}^{\prime}=-G \theta_{011} \\
& u_{012}^{\prime \prime}+u_{012}^{\prime}=-G \theta_{012} \\
& u_{021}^{\prime \prime}+u_{021}^{\prime}=-G \theta_{021}+u_{011}^{\prime \prime \prime} \\
& u_{022}^{\prime \prime}+u_{022}^{\prime}=-G \theta_{022}+u_{012}^{\prime \prime \prime} \\
& u_{111}^{\prime \prime}+u_{111}^{\prime}-i n u_{111}=-i n-G \theta_{111} \\
& u_{112}^{\prime \prime}+u_{112}^{\prime}-i n u_{112}=-G \theta_{112} \\
& u_{121}^{\prime \prime}+u_{121}^{\prime}-i n u_{121}=-G \theta_{121}+u_{111}^{\prime \prime \prime}+i n u_{111}^{\prime \prime} \\
& u_{122}^{\prime \prime}+u_{122}^{\prime}-i n u_{122}=-G \theta_{122}+u_{112}^{\prime \prime \prime}+i n u_{112}^{\prime \prime} \\
& \theta_{011}^{\prime \prime}+P \theta_{011}^{\prime}-P \phi \theta_{011}=0 \\
& \theta_{012}^{\prime \prime}+P \theta_{012}^{\prime}-P \phi \theta_{012}=-P u_{011}^{\prime 2} \\
& \theta_{021}^{\prime \prime}+P \theta_{021}^{\prime}-P \phi \theta_{021}=0 \\
& \theta_{022}^{\prime \prime}+P \theta_{022}^{\prime}-P \phi \theta_{022}=-2 P u_{011}^{\prime} u_{021}^{\prime}
\end{aligned}
$$

$$
\begin{aligned}
& \theta_{111}^{\prime \prime}+P \theta_{111}^{\prime}-(i n-\phi) P \theta_{111}=0 \\
& \theta_{112}^{\prime \prime}+P \theta_{112}^{\prime}-(i n-\phi) P \theta_{112}=-2 P u_{011}^{\prime} u_{111}^{\prime} \\
& \theta_{121}^{\prime \prime}+P \theta_{121}^{\prime}-(i n-\phi) P \theta_{121}=0 \\
& \theta_{122}^{\prime \prime}+P \theta_{122}^{\prime}-(i n-\phi) P \theta_{122}= \\
& \quad-2 P\left(u_{011}^{\prime} u_{121}^{\prime}+u_{021}^{\prime} u_{111}^{\prime}\right)
\end{aligned}
$$

and the corresponding boundary conditions are

$$
\left.\begin{array}{rl}
y=0 & u_{011}=0, u_{012}=u_{111}=u_{112}=0 \\
& \theta_{011}=-1, \theta_{012}=\theta_{111}=\theta_{112}=0
\end{array}\right\}
$$

where dashes denotes differentiation with respect to $y$

$$
\left.y \rightarrow \infty \quad \begin{array}{c}
u_{011} \rightarrow 1, u_{012}=u_{112} \rightarrow 0, u_{111} \rightarrow 1 \\
\theta_{011} \rightarrow 0, \theta_{012} \rightarrow \theta_{111} \rightarrow \theta_{112} \rightarrow 0
\end{array}\right\}
$$

The solutions of the above mentioned differential equations consistent with the boundary conditions (43) for unsteady flow field have been obtained but not presented here for the sake of brevity.

With the convection those real parts of complex number have physical significance in the problem, the velocity and temperature field can be expressed in fluctuating part as

$$
\begin{aligned}
& u(y, t)=u_{0}(y)+\varepsilon\left(M_{r} \cos n t-M_{i} \sin n t\right) \\
& \theta(y, t)=\theta_{0}(y)+\varepsilon\left(T_{r} \cos n t-T_{i} \sin n t\right)
\end{aligned}
$$

where

$$
\left.\begin{array}{l}
M_{r}+i M_{i}=u_{1} \\
T_{r}+i T_{i}=\theta_{1}
\end{array}\right\}
$$

Hence expressions for transient velocity, temperature field for $n t=\frac{\pi}{2}$ are

$$
u\left(y, \frac{\pi}{2 n}\right)=u_{0}-\varepsilon M_{i}
$$


$\theta\left(y, \frac{\pi}{2 n}\right)=\theta_{0}-\varepsilon T_{i}$

Skin friction

Skin friction $(\tau)$ at plate, in terms of amplitude and phase is

$\tau=\left(\frac{\partial u}{\partial y}\right)_{y=0}=\tau_{0}+\varepsilon|N| \cos (n t+\alpha)$

where

$N=N_{r}+i N_{r}=\left(\frac{\partial u_{1}}{\partial y}\right)_{y=0}$

and phase angle $\tan \alpha=\frac{M_{i}}{M_{r}}$

The expressions (46) - (47) and (48) - (49) will be used for numerical calculation of the velocity, temperature field and for the skin friction and phase of the skin friction respectively.

\section{RESULT AND DISCUSSIONS}

The purpose of this study is to bring out the effects of elasticoviscous parameter and heat absorption parameter on the free convective flow and heat transfer characteristics. The elasticoviscous effect is exhibited through the non-dimensional parameter $\mathrm{k}$. The corresponding results for Newtonian fluid can be deduced from the above results by setting $\mathrm{k}=0$ and it is worth mentioning here that these results coincide with that of Ahmed et. al. Numerical evaluation of analytical results reported in the previous section was performed and a representative set of results is reported graphically for two cases viz. (i) for cooling of the plate $(G>0)$ and (ii) for heating of the plate $(\mathrm{G}<0)$. The obtained results are illustrated in Figs. 1 to 14. The values of $\mathrm{P}$ (Prandatl number) are chosen in such a way that it represent Gasoline at $20^{\circ} \mathrm{C}(\mathrm{P}=9.4)$ and water at $4^{\circ} \mathrm{C}(\mathrm{P}=11.13)$, whereas $\mathrm{E}$ is taken small for incompressible fluid.

For the case of different values of thermal Grashofnumber $G$, the mean velocity profiles are shown in figure 1. As expected, it is observed that an increase in $G$ leads to rise in the values of velocity due to enhancement in boundary force. In addition the curve show that the peak values of the velocity increases rapidly near the plate and there after it decreases as distance (y) increases. It is also observed that the mean velocity of the elastic-viscous fluid is greater than the Newtonian fluid. Figures 2 and 3 are plotted mean velocity profiles against y for different values of heat absorption parameter $\phi$. It is observed that in both the cases mean velocity of the elastico - viscous fluid is less than that of Newtonian fluid. The maximum velocity occurs near the plate and thereafter it decreases as distance (y) increases. An increase in $\phi$ leads to decrease in the mean velocity. From figure 4 it is observed that mean velocity of the elastico - viscous fluid is greater than that of Newtonian fluid. The maximum velocity occurs near the plate and thereafter it decreases as distance (y) increases. An increase in $E$ the mean velocity increases. Figures(5)-(11) represent the temperature profiles for $G>0$ and $G<0$, respectively for different values of Eckret number (E), Grashof number $(\mathrm{G})$, Prandtl number $(\mathrm{P})$ and heat absorption number $(\phi)$. It is observed that for cooling of the plate $(G>$ $0)$, an increase in the above said parameters decreases the temperature. The increase of Prandtl number results in the decrease of temperature distribution. This is due to the fact that there would be a decrease of thermal boundary layer thickness with the increase of Prandtl number. In the case of heating of the plate i.e. for $G<0$, an increase in $E$ leads to fall in the temperature (fiure-9). It is also concluded that for electrolytic solution, temperature falls exponentially. While reversal effect is observed (fig10 and 11) for $\mathrm{P}$ and $\phi$. It is observed from figure 12 that the mean velocity of the elastico - viscous fluid is greater than that of Newtonian fluid. The maximum velocity occurs near the plate and thereafter it decreases as distance (y) increases. An increase in Prandtl number $P$ the mean velocity decreases. Figure 13 gives the mean velocity profile for heating of the plate $(G<0, E<0)$. From this we conclude that mean velocity of the Newtonian fluid is less than that of elastico - viscous fluid. The minimum value of the velocity occurs near the plate and it increases as the $y$ increases. Velocity distribution decreases as E increases. Figure 14 gives the mean velocity profile for heating of the plate $(G<0, E<0)$.From this we conclude that mean velocity of the Newtonian fluid is greater than that of elastico - viscous fluid. The minimum value of the velocity occurs near the plate and it increases as the $y$ increases. The velocity profiles decreases with increasing Prandtl number. Physically, this is true because the increase in the Prandtl number is due to increase in the viscosity of the fluid which makes the fluid thick and hence a decrease in the velocity of the fluid.

\section{ACKNOWLEDGEMNETS}

The authors are highly thankful to Sri Venkateswara University, Tirupati, AP, Inida to provide all the facilities to do this research work.

\section{REFERENCES}

[1] Beg, O.A., Takhar, H.S., and Singh, A.K., Multiparameter Perturbation Analysis of Unsteady 
Oscillatory Magneto-convection in Porous Media with Heat Source Effects, International Journal of Fluid Mech. Research, 32,(2005), pp. 635-661.

[2] Burak,V.S, Volkov, S.V, Martyneko,O.G, Kharmastov, P.P and Sikh, I.A, Free convection heat transfer on a vertical surface with heat flux discontinuity, Int.J.Heat Mass Trans. Vol. 38 (1) (1995), p. 155.

[3] Chaudhary, R.C,.andAbhay Kumar Jha, Free convection oscillatory flow of an elastico-viscous fluid past an impulsively started infinite vertical plate with constant heat flux, Far East Journal of Applied Mathematics.31 (2008), pp. 337-352.

[4] Chenna Kesavaiah D and Sudhakaraiah A: A note on Heat Transfer to Magnetic Field Oscillatory Flow of a Viscoelastic Fluid, International Journal of Science, Engineering and Technology Research (IJSETR), Vol. 2, (5), 2013, pp. 1007-1012

[5] Devika B, SatyaNarayana P. V and Venkataramana S: MHD Oscillatory Flow of a Viscoelastic Fluid in a Porous Channel with Chemical Reaction, International Journal of Engineering Science Invention, Vol. 2 (2), 2013, pp.26-35

[6] Erickson, L.E., Fan, L.T. and Fox, V.G.: Heat and mass transfer on a moving continuous flat plate with suction or injection, Ind. Engg. Chem. Fund.,(1966), 5, 19.

[7] Hayat.T, Mohyuddin, M.R and Aghar. S: Flow of viscoelastic fluid on an oscillating plate, ZAMM 84 (1) (2004), p. 65.

[8] Kolani, P.N: Phys. Fluids, 10,( 1967), p. 1344.

[9] Lee. S and Yovanovich.M.M, Linearization of natural convection from a vertical plate with arbitrary heat flux distribution, J.Heat Trans, 144 (1992), p. 909.

[10] Lighthill, M.J., The response of laminar skin friction and heat transfer to fluctuation in the stream velocity, Proc.Soc.London Ser.A 224, (1954), 1.

[11] Malarvizhi. G, Ramanaish. G, and Pop.I, Free and mixed convection about a vertical plate with prescribed temperature or heat flux, ZAMM 74 (1994), p. 129.

[12] Pantokraters. A, Laminar free convection in water with variable physical properties adjacent to vertical plate with uniform heat flux, Int.J.Heat Mass Trans. 46 (2003), p. 725.

[13] Revankar. S.T, Free convection effects on a flow past an impulsively started or oscillating infinite vertical plate, Mech.Research Comm. 27 (1981), p. 241.

[14] Sakiadis, B.C.: Boundary layer behavior on continuous solid surface, A 1 ch E J. (1961), 7, 26.

[15] Sharma, P.R. and Gocher, D.K.: J. Ultra Scientist Phyl. Sciences, India, (1998), 11, p. 312.

[16] Singh A.K, Oscillating free convection flow of an elastico-viscous flow past an impulsively started infinite vertical plate - I, Ind.J.Tech. 27, (1984), 245.

[17] Singh K. D: "Exact solution of an oscillatory MHD flow in a channel filled with porous medium", Int. J. of Applied Mechanics and Engineering, Vol. 16, pp.277283 (2011).
[18] Soundalgerkar V.M, Unsteady free convection flow of an elastico - viscous fluid past an infinite vertical plate with constant suction. Chem.Engg.Sci. 26 (1971), p. 2043.

[19] SudheerBabu. M and SatyaNarayana. P.V., Effects of the chemical reaction and radiation absorption on free convection flow through porous medium with variable suction in the presence of uniform magnetic field, J.P. Journal of Heat and mass transfer,Vol. 3 (3), (2009), pp.219-234.

[20] Tsou, F., Sparrow, E.M. and Goldstein, R.: Int. J. Heat Mass Transfer,(1967) 10, p. 219.

[21] Vajravelu, K :ZAMM,(1987), 8, p. 67.

[22] Walters. K, The solution of flow problem in the case of materials with memory J.Mech. 1 (1962), p. 474.

\section{BIOGRAPHIES:}

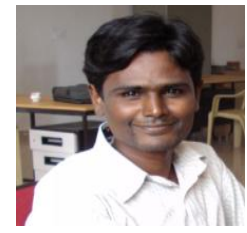

D Chenna Kesavaiah: Received B. Sc from Nagarjuna University, Guntur, M. Sc from Jawaharlal Nehru Technological University College of Engineering, Hyderabad, Ph. D from Sri Venkateswara University, Tirupati, AP, India, and working as Sr. Asst. Professor in Visvesvaraya College of Engineering \& Technology, Greater Hyderabad, Andhra Pradesh, India.

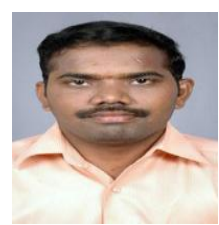

P.V. Satyanarayana: Received Graduation from Silver Jubilee Degree college, Kurnool. He completed M.Sc., M.Phil., Ph.D. in Mathematics from Sri Venkateswara University, Tirupati. Presently he is working as Assistant Professor (Senior) of Mathematics at VIT University, Vellore, TN, India.

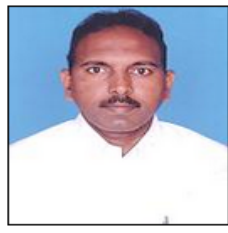

A Sudhakaraiah: Received B. Sc, M. Sc, Ph. D from Sri Venkateswara University, Tirupati, and Working as Assistant Professor, Department of Future studies, Sri Venkateswara University, Tirupati, Andhra Pradesh, India.

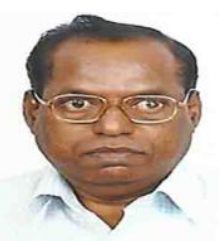

S. Venkataramana Received M. Sc., M. Phil and $\mathrm{Ph} \mathrm{D}$ from Sri Venkateswara University, Tirupati, AP, India and working as Professor in Sri Venkateswara University, Tirupati, AP, India. 


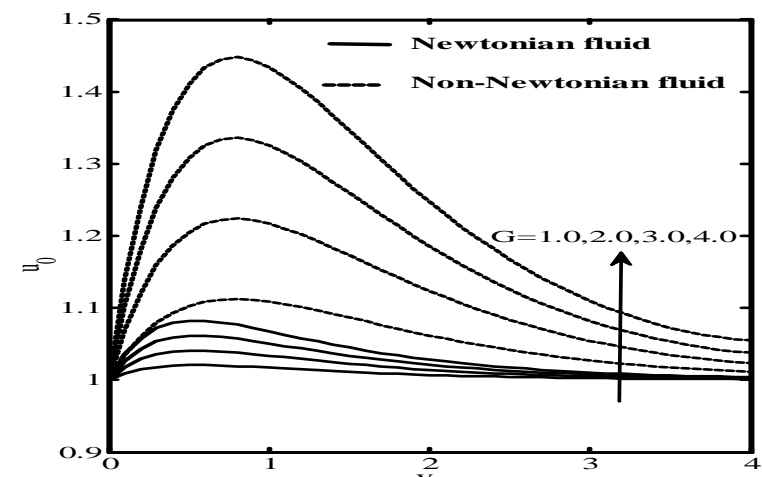

Fig 1.Mean velocity profiles for cooling of the plate $E=0.01$

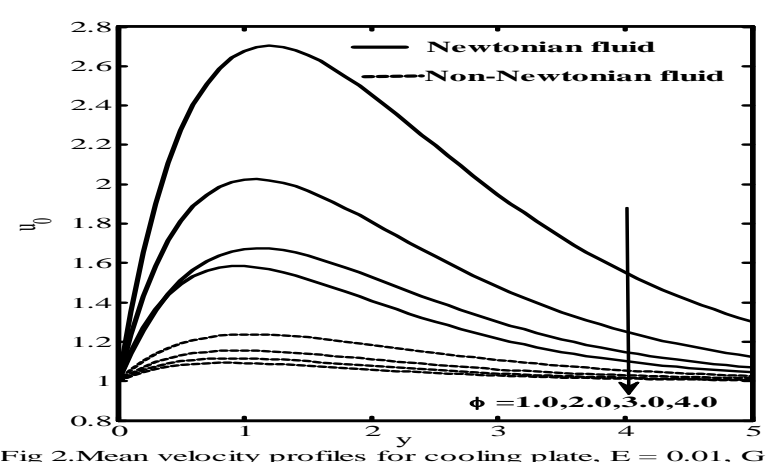

Fig 2.Mean velocity profiles för cooling plate, $E=0.01, G>0$

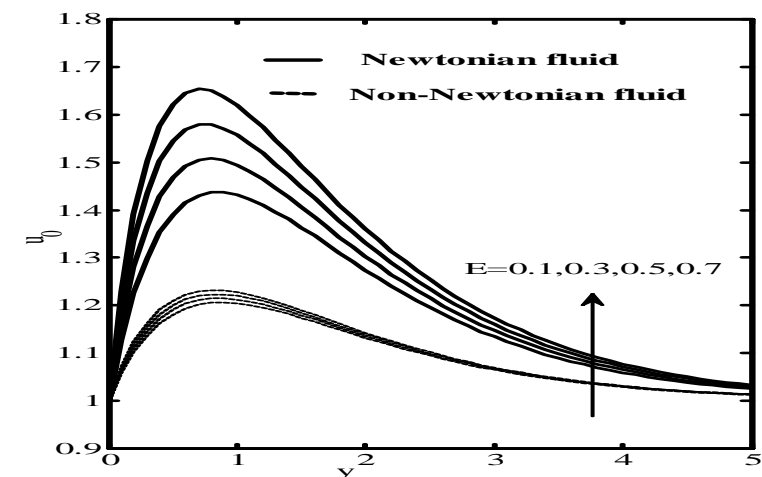

Fig 3. Mean velocity profile of the cooling plate, $G>0$

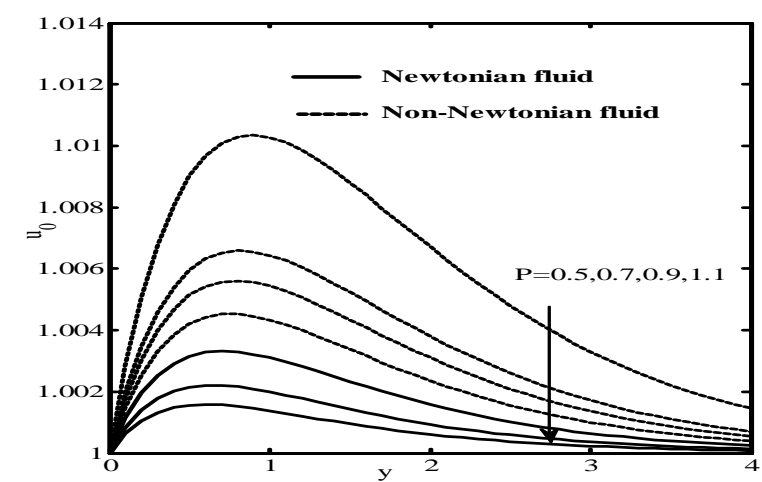

Fig 4. Mean velocity profiles cooling of the plate, $E=0.01, G>0$
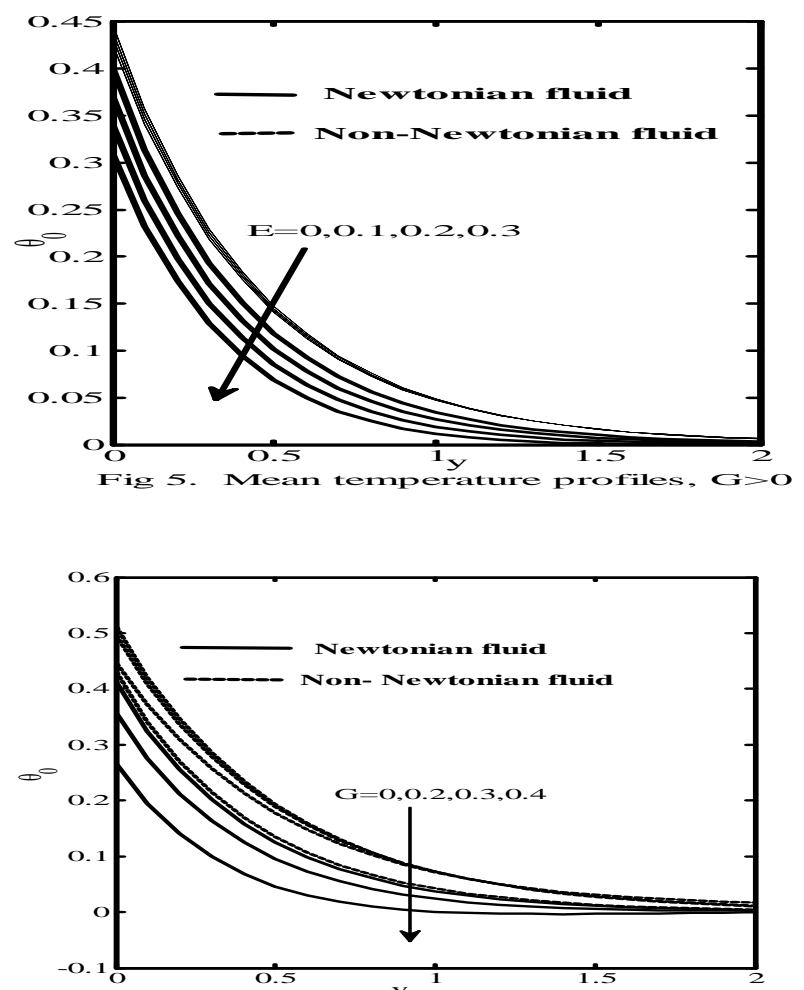

Fig 6. Mean temperature profiles cooling of the plate $E=0.01$

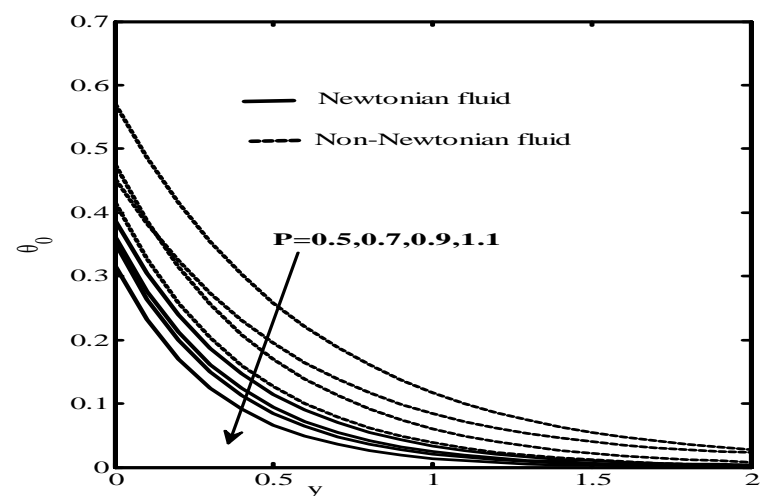

Fig 7.Mean temperature profiles cooling of the plate $\mathrm{E}=0.01, \mathrm{G}>0$

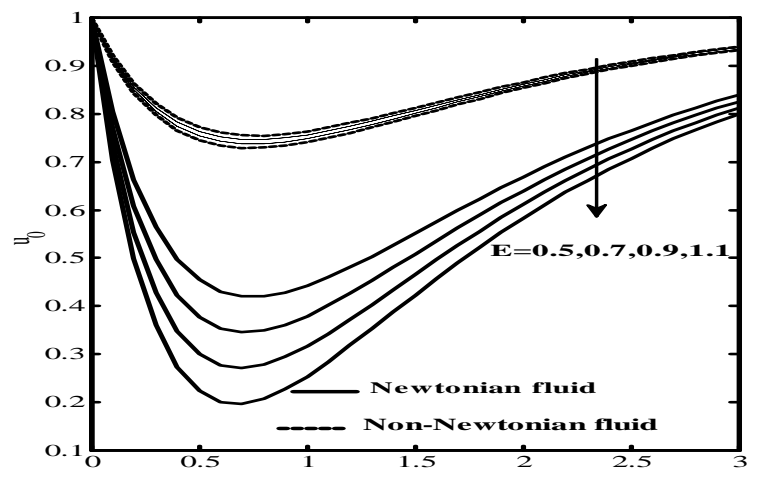

Fig 9.Mean velocity for heating of the plate $\mathrm{E}=-0.01, \mathrm{G}<\mathrm{O}$ 


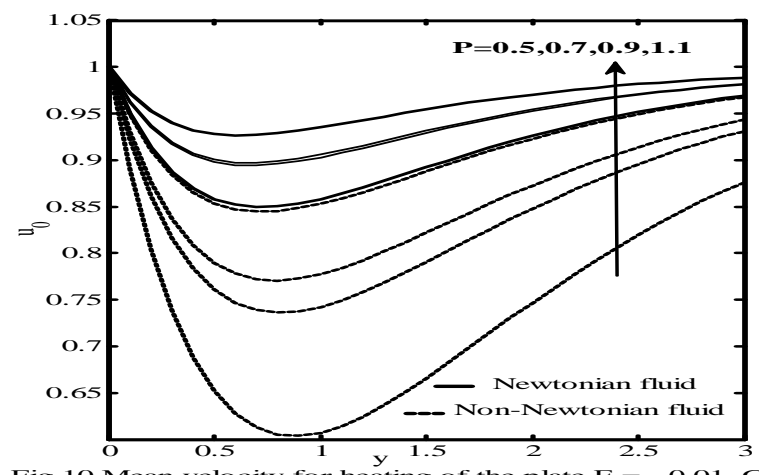

Fig 10. Mean velocity for heating of the plate $\mathrm{E}=-0.01, \mathrm{G}<\mathrm{O}$

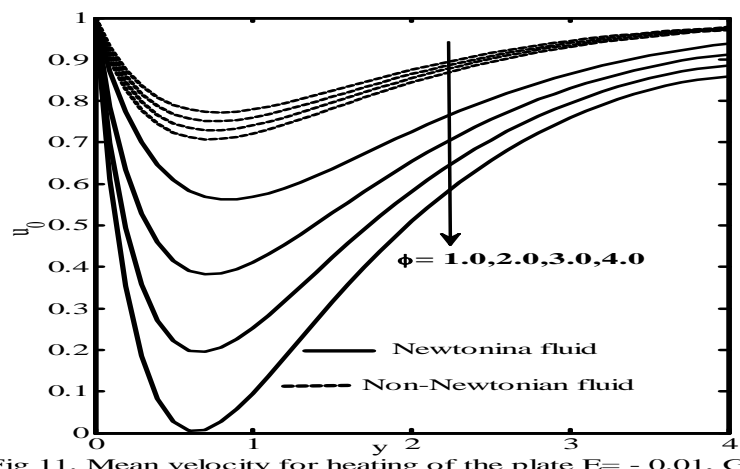

Fig 11. Mean velocity for heating of the plate $\mathrm{E}=-0.01, \stackrel{4}{\mathrm{G}}<0$

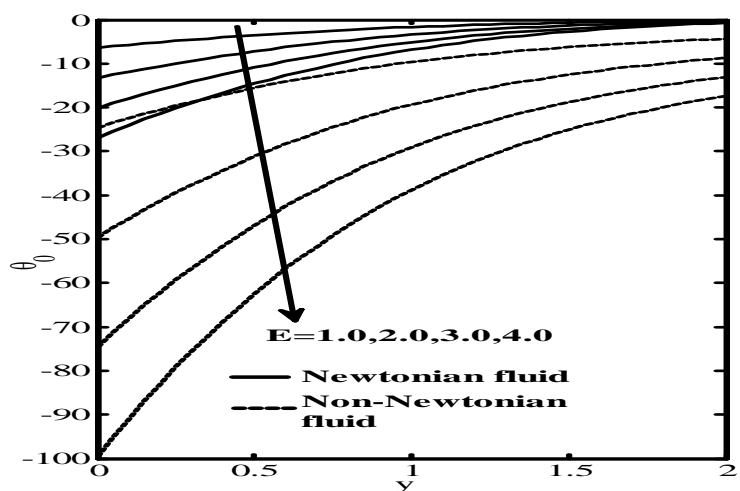

Fig 12. Mean temperature heating of the plate, $G<0$

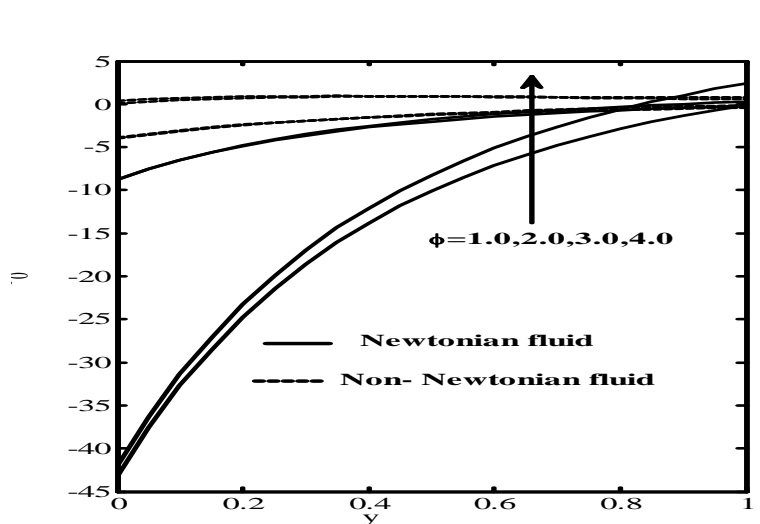

Fig 13. Mean temperature heating of the plate $\mathrm{E}=-\mathbf{0 . 0 1}, \mathrm{G}<\mathrm{O}$

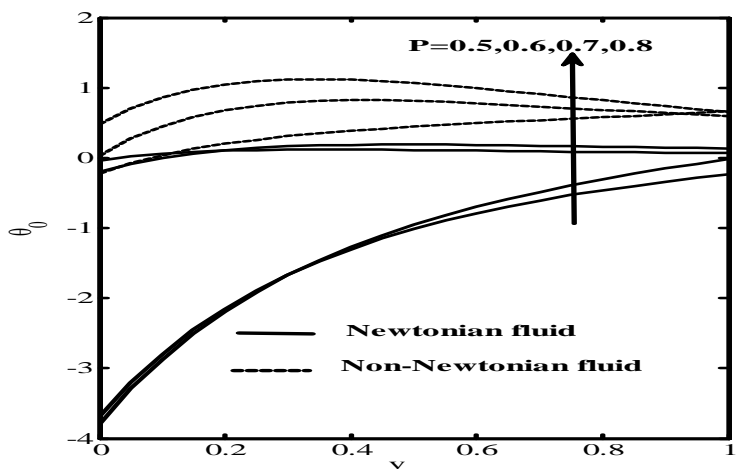

Fig 14 Mean temperature heating of the plate $E=-0.01, G<0$ 